\title{
Tumour Markers in the Differential Diagnosis of Patients With Isolated Involuntary Weight Loss
}

\author{
JAUME TRAPE ${ }^{1,2}$, JORDI ALIGUE ${ }^{2,3,4}$, MIREIA VICENTE ${ }^{5}$, ANNA ARNAU ${ }^{6}$, ANTONIO SAN-JOSE ${ }^{7}$, \\ JOSEP ORDEIG ${ }^{4}$, ROSER ORDEIG $^{4}$, MARIONA BONET $^{4}$, ANDRES ABRIL ${ }^{4}$, OMAR EL-BOUTROUKI $^{4}$, \\ CAROLINA GONZALEZ-FERNANDEZ ${ }^{1}$, MARIA SALA ${ }^{1}$, CRISTINA FIGOLS ${ }^{1}$, \\ ELISABETH GONZALEZ-GARCIA ${ }^{5}$, LOURDES MONTSANT ${ }^{1}$ and DOMINGO RUIZ ${ }^{4}$ \\ ${ }^{1}$ Laboratory Medicine Althaia, Xarxa Assistencial Universitària de Manresa, Manresa, Spain; \\ ${ }^{2}$ Faculty of Medicine, Universitat de Vic, Universitat Central de Catalunya, Vic, Spain; \\ ${ }^{3}$ Central Catalonia Chronicity Research Group $(C 3 R G)$, Internal Medicine Althaia, \\ Xarxa Assistencial Universitària de Manresa, Manresa, Spain; \\ ${ }^{4}$ Internal Medicine Althaia, Xarxa Assistencial Universitària de Manresa, Manresa, Spain; \\ ${ }^{5}$ CAP Manresa 2 Institut Catala de la Salut Barcelona, Barcelona, Spain; \\ ${ }^{6}$ Clinical Research Unit, Althaia Xarxa Assistencial Universitària de Manresa, Manresa, Spain; \\ ${ }^{7}$ Internal Medicine, Vall d'Hebron University Hospital, Barcelona, Spain
}

\begin{abstract}
Background/Aim: Paraneoplastic syndrome symptoms include isolated involuntary weight loss (IIWL). The differential diagnosis of cancer from other diseases may require a significant number of tests. Tumour markers (TMs) can be used for the diagnosis and stratification of patients according to cancer risk. Patients and Methods: This study included 606 patients (48\% females) seen at the rapid diagnostic unit for IIWL. We determined the levels of TMs carcinoembryonic antigen, carbohydrate antigen 19-9, soluble fragments of cytokeratin 19, carbohydrate antigen 15-3, carbohydrate antigen 125, neuron specific enolase, alpha-fetoprotein, prostatic specific antigen using the multiparametric analyser COBAS 601. Two cut-off points were established, the upper reference limit described by the manufacturer and a high cut-off point suggested by Molina et al., to stratify patients according to cancer risk. Results: Patients were classified according to TM levels as follows: I) all TMs below the upper reference limit; II) highest number of TMs between the two cut-offs; III) at least one TM above the higher cut-off. The odds ratio for malignancy was
\end{abstract}

This article is freely accessible online.

Correspondence to: Jaume Trapé, PhD, Laboratory Medicine, Althaia Xarxa Assistencial Universitària de Manresa, Dr Joan Soler 1-3, 08243 Manresa, Catalonia, Spain. Tel: +34 938742112, e-mail: jtrape@althaia.cat

Key Words: Weight loss, tumour markers, differential diagnosis, cancer, CEA, CYFRA 21-1.
4.3 for group II and 248 for group III. These results indicate that when at least one TM is above the higher cut-off, neoplasia is highly probable. Conclusion: TM determination allowed to establish cancer risk in patients with IIWL.

Many cancer patients do not present specific symptoms or signs when they consult medical services $(1,2)$. These symptoms, including cachexia, weight loss, and muscle wasting arise from tumour secretion of hormones, peptides or cytokines $(3,4)$ or in case of autoimmune neurological disorders, from immune cross reactivity between malignant and normal host tissues $(5,6)$. Other disorders are due to tumour invasion or compression in surrounding or distant tissues.

In some cases, paraneoplastic syndromes are presented before cancer diagnosis and may be used to select patients for more exhaustive tests, in order to perform differential diagnosis with benign diseases.

In our country, specialised hospital units have been created to avoid unnecessary delays for patients with these symptoms. The rapid diagnostic unit (RDU) is an ambulatory resource for the study of severe pathologies without the need for hospital admission. It refers to patients with guidesymptoms derived from other hospital services or primary care. Some of the frequent signs that require referral to RDU are constitutional syndrome, wasting syndrome, or isolated involuntary weight loss (IIWL). Between 10 and $30 \%$ of patients who arrive at these units present involuntary weight loss as the only sign. In the RDU, they are being prioritized to undergo endoscopies, image tests, blood tests and biopsies in order to reach a diagnosis and start treatment when possible within one month (7-9). 


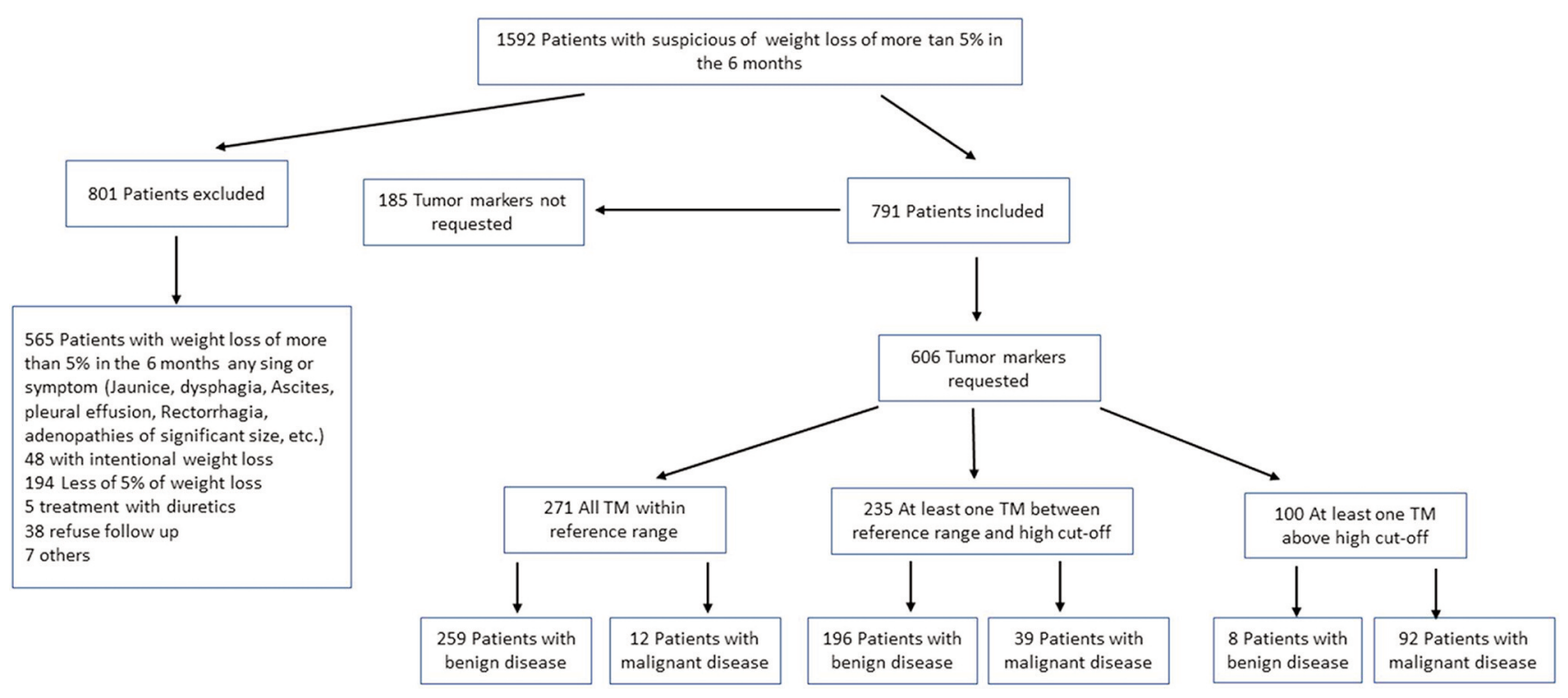

Figure 1. Flow chart.

As these signs or symptoms are not specific to cancer, in patients with constitutional syndrome, wasting syndrome or IIWL, a differential diagnosis must be made between different types of cancers such as lung cancer, lymphoma, renal cancer, and benign diseases such as tuberculosis, toxoplasmosis, pneumonia, and chronic intestinal infections (1).

TM measurement can aid diagnosis and serve as a complement to expensive imaging techniques and invasive testing (10-15). However, increased plasma levels of TMs may also be observed in patients without cancer due to a variety of processes, and thus give rise to false positives (16).

The aim of our study was to determine the diagnostic accuracy of TMs in the differential diagnosis of patients with IIWL and their ability to predict the risk of cancer.

\section{Patients and Methods}

This is an observational study including patients seen for IIWL at the RDU between January 2005 and December 2013. The followup of the last patient was completed in December 2014.

At the discretion of the attending physician, analytical parameters including TMs were obtained from patients with IIWL referred from primary or emergency care to the RDU. All patients presenting documented idiopathic IIWL of at least 5\% over the past 12 months were included in the study. IWL was considered isolated when it was not accompanied by symptoms or signs specific of an organ or system. When weight loss was not documented, the criteria of Marton et al. were used (17), according to which patients were eligible if they met at least two of the following criteria: evidence of change in clothes size, confirmation of weight loss by a friend or relative, and ability to give a numerical estimate of weight loss. Exclusion criteria were as follows: (i) specific symptoms (jaundice, ascites, dysphagia for at least 6 months, diarrhoea for at least 6 months, rectorrhagia, serious rhythm deposition changes, rectal tenesmus and/or suspicious rectal examination, subocclusive crises, change in cough for at least one month, haemoptysis of unknown origin, dysphonia for at least one month, palpable breast mass, nipple discharge, macroscopic haematuria, significant increase in lymph nodes volume, (>1 cm) and malignant hepatomegaly); (ii) intentional weight loss; (iii) start of diuretic treatment three months before start of IWL; (iv) weight loss of $<5 \%$ or no weight loss observed during first visit at RDU; (v) refusal to participate in follow-up assessments and/or undergo further complementary tests; (vi) non-compliance with study criteria for RDU; and (vii) death during the diagnostic process.

The RDU database was used to identify patients. Demographic data, clinical and follow-up history were obtained from patients' clinical records. The study protocol was approved on March 6, 2015 by an independent clinical research ethics committee (Comitè d’Ètica d'Investigació Clínica de la Fundació Unió Catalana d'Hospitals) (Ethics Committee number CEIC 15/16).

All TMs were measured on the day of the first visit: carcinoembryonic antigen (CEA), carbohydrate antigen CA19-9 (CA19-9), soluble fragments of cytokeratin 19 (CYFRA 21-1), carbohydrate antigen CA15-3 (CA15-3), carbohydrate antigen 125 (CA125), neuron specific enolase (NSE), alpha-fetoprotein (AFP), prostatic specific antigen (PSA) in a multiparametric analyser COBAS 601 by an electrochemiluminescent assay (Roche Diagnostics, Sant Cugat, Barcelona, Spain). Upper reference limit was that described by the manufacturer for CEA, CA19-9, CYFRA 21-1, CA15-3, CA125, NSE, AFP and PSA were $5 \mu \mathrm{g} / 1,37 \mathrm{U} / \mathrm{ml}$, $3.3 \mu \mathrm{g} / 1,30 \mathrm{U} / \mathrm{ml}, 35 \mathrm{U} / \mathrm{ml}, 17 \mu \mathrm{g} / 1,10 \mu \mathrm{g} / \mathrm{l}$ and $4 \mu \mathrm{g} / 1$ respectively. Samples in which NSE determinations had been requested were visually inspected and those that showed any orange coloration suspected of minimal haemolysis were excluded. When NSE values were above the upper reference limit, another visual verification of haemolysis was carried out, and in the case of minimum orange coloration the result was invalidated. The cut-off values described 
by Molina et al. (18) were used for patients without hepatopathy and renal failure: $15 \mu \mathrm{g} / 1,7.8 \mu \mathrm{g} / \mathrm{l}, 200 \mathrm{U} / \mathrm{ml}, 45 \mu \mathrm{g} / \mathrm{l}, 40 \mu \mathrm{g} / 1,30$ $\mu \mathrm{g} / \mathrm{l}$, and $100 \mathrm{U} / \mathrm{ml}$ for CEA, CYFRA 21-1, CA19-9, NSE, AFP, PSA, and CA15-3 respectively. Patients were classified into three groups, group I: those with all TMs below the reference limit; group II: those with at least one TM between the upper reference limit and below the cut-off proposed by Molina et al. (18); and group III: those with at least one TM above the cut-off proposed by Molina et al. (18). To obtain a definitive diagnosis, serological testing, and culture and image analysis were required. The diagnosis of cancer was established by histological study of biopsies obtained by gastroscopy, colonoscopy, bronchoscopy, laparoscopy, thoracoscopy or direct study of the tumour obtained by tumorectomy.

Statistical analysis. Sensitivity, specificity, positive predictive value (PPV), negative predictive value (NPV), positive likelihood ratio (PLR) and negative likelihood ratio (NLR) were calculated for each TM and for the combination of all TMs using the two cut-off points. The parameters of diagnostic accuracy are shown together with their $95 \%$ confidence interval $(95 \% \mathrm{CI})$.

A two-sided 5\% significance level was assumed. All statistical analyses were performed using IBM $^{\circledR}$ SPSS $^{\circledR}$ Statistics for Windows v.22 (IBM Corporation ${ }^{\circledR}$, Armonk, NY, USA) and Stata ${ }^{\circledR}$ v.14 (StataCorp LP ${ }^{\circledR}$, College Station, TX, USA).

\section{Results}

A total of 606 patients with IIWL were analysed, aged between 23 and 95 years, mean 69 years (SD 14.1), and 194 $(48 \%)$ were women. Figure 1 shows the flow chart of the study. Table I shows the final diagnosis: $23.6 \%$ had cancer (42.7\% digestive, $17.5 \%$ lung, and $11.9 \%$ non-epithelial), $30.9 \%$ psychiatric disorders (mainly depressive syndrome), $42.2 \%$ non-neoplastic organic disease and $3.3 \%$ idiopathic IIWL. Ninety-two cancer patients $(64.3 \%)$ had metastatic disease and $37(25.9 \%)$ had stage IIIA or IIIB. Table II shows tumour marker concentrations according to disease type in the final diagnosis.

Table III and Table IV show the sensitivity, specificity, PPV, NPV, PLR and NLR ratios for TM at two different cutoffs for CEA, CA125, CYFRA 21-1, CA15-3, CA19-9, NSE, AFP and PSA. Sensitivity and NPV were $91.6 \%$ and $95.7 \%$, respectively (Table III) when the upper reference limit was used as cut-off. A higher specificity (98.3\%) and PPV $(92.0 \%)$ were obtained at the cut-off proposed by Molina et al. (18) (Table IV). Figure 2 shows tumour prevalence, stage (metastatic $v s$. no metastatic), epithelial $v s$. non-epithelial, type of benign disease, and odds ratio for cancer according to each group.

\section{Discussion}

Our study evaluated the performance of TMs to differentiate between neoplasia and benign disease in 606 patients with involuntary isolated weight loss as the only sign suggestive of cancer. Regarding diagnostic performance, using the high
Table I. Final diagnosis of patients with unintentional weight loss included in the study.

\begin{tabular}{|c|c|c|c|}
\hline Aetiology & Percent & Age (years) & $\%$ Women \\
\hline Neoplasia & $143(23.6 \%)$ & $71.2 \pm 11.5$ & 31.2 \\
\hline Epithelial & $126(20.8 \%)$ & & \\
\hline Lung cancer & $25(4.1 \%)$ & & \\
\hline Stomach & $22(3.6 \%)$ & & \\
\hline Colorectal & $21(3.5 \%)$ & & \\
\hline Pancreas & $18(3.0 \%)$ & & \\
\hline $\begin{array}{l}\text { Cancer of unknown } \\
\text { primary }\end{array}$ & $11(1.8 \%)$ & & \\
\hline Kidney and urinary tract & $10(1.7 \%)$ & & \\
\hline Liver and bile ducts & $6(1.0 \%)$ & & \\
\hline Prostate & $3(0.5 \%)$ & & \\
\hline Breast & $3(0.5 \%)$ & & \\
\hline Others & $7(1,2 \%)$ & & \\
\hline Non epithelial & $17(2.8 \%)$ & & \\
\hline Lymphoma & $6(1.0 \%)$ & & \\
\hline Multiple myeloma & $5(0.8 \%)$ & & \\
\hline Leukaemia & $4(0.7 \%)$ & & \\
\hline Leiomyosarcoma & $2(0.3 \%)$ & & \\
\hline $\begin{array}{l}\text { Myelodyosplasia } \\
\text { syndrome }\end{array}$ & $1(0.2 \%)$ & & \\
\hline Organic no neoplastic & $256(42.2 \%)$ & $69.3 \pm 14.7$ & 47.9 \\
\hline Digestive system & $100(16.5 \%)$ & & \\
\hline Peptic disorders & $35(5.8 \%)$ & & \\
\hline Hiatus hernia & $15(2.8 \%)$ & & \\
\hline $\begin{array}{l}\text { Chronic alcohol induced } \\
\text { liver disease }\end{array}$ & $13(2.1 \%)$ & & \\
\hline Gallstones & $7(1.2 \%)$ & & \\
\hline Functional disorders & $5(0.8 \%)$ & & \\
\hline Barrett's esophagus & $3(0.5 \%)$ & & \\
\hline $\begin{array}{l}\text { Chronic alcohol induced } \\
\text { pancreatitis }\end{array}$ & $3(0.5 \%)$ & & \\
\hline Chronic Hepatitis C & $2(0.3 \%)$ & & \\
\hline Benign colon polyps & $2(0.3 \%)$ & & \\
\hline Other disorders & $15(2.8 \%)$ & & \\
\hline Pharmacological causes & $37(6.1 \%)$ & & \\
\hline Digoxin & $8(1.3 \%)$ & & \\
\hline Metformin & $7(1.2 \%)$ & & \\
\hline Opioids & $5(0.8 \%)$ & & \\
\hline $\begin{array}{l}\text { Non-steroidal } \\
\text { anti-inflammatories }\end{array}$ & $4(0.7 \%)$ & & \\
\hline Levothyroxine & $4(0.7 \%)$ & & \\
\hline Others & $9(1.5 \%)$ & & \\
\hline Infections & $23(3.8 \%)$ & & \\
\hline Rheumatism & $20(3.3 \%)$ & & \\
\hline Neurological disorders & $20(3.3 \%)$ & & \\
\hline Endocrine disorders & $11(1.8 \%)$ & & \\
\hline Lung disorders & $10(1.7 \%)$ & & \\
\hline Musculoskeletal disorders & $10(1.7 \%)$ & & \\
\hline Heart disorders & $8(1.3 \%)$ & & \\
\hline Psychosocial circumstances & $7(1.2 \%)$ & & \\
\hline Active alcoholism & $3(0.5 \%)$ & & \\
\hline Others & $7(1.2 \%)$ & & \\
\hline Psychiatric disorders & $187(30.9 \%)$ & $67.6 \pm 15.8$ & 64.5 \\
\hline Depression & $16(26.7 \%)$ & & \\
\hline Anxiety & $15(2.5 \%)$ & & \\
\hline Eating disorders & $3(0.5 \%)$ & & \\
\hline Others & $7(1.2 \%)$ & & \\
\hline Unknown origin & $20(3.3 \%)$ & $62.1 \pm 20.5$ & 45.0 \\
\hline
\end{tabular}


in vivo $35: 3361-3367(2021)$

Table II. Serum concentrations of tumours markers according to final diagnosis.

\begin{tabular}{|c|c|c|c|c|c|c|c|}
\hline & & \multicolumn{3}{|c|}{ Neoplasia } & \multicolumn{3}{|c|}{ Benign diseases } \\
\hline & & $\underset{(n=143)}{\operatorname{All}} \quad \mathrm{N}$ & $\begin{array}{l}\text { Neoplasia epithelial } \\
\qquad(n=126)\end{array}$ & $\begin{array}{c}\text { Neoplasia } \\
\text { non-epithelial } \\
(n=17)\end{array}$ & $\begin{array}{c}\text { Organic } \\
\text { no neoplastic } \\
(\mathrm{n}=256)\end{array}$ & $\begin{array}{l}\text { Psychiatric } \\
(\mathrm{n}=187)\end{array}$ & $\begin{array}{c}\text { Unknown } \\
\text { origin } \\
(\mathrm{n}=20)\end{array}$ \\
\hline \multirow[t]{2}{*}{ CEA $(\mu \mathrm{g} / 1)$} & Mean (SD) & $94.6(355.6)$ & 107 (377) & $2.1(1.1)$ & $3.1(2.7)$ & $2.7(2.2)$ & $2.1(1.95)$ \\
\hline & Median (IQR) & $5(20.9)^{\mathrm{a}}$ & $6.6(30.0)^{\mathrm{b}, \mathrm{c}}$ & $1.8(2.0)$ & $2.2(2.4)$ & $2.2(1,7)$ & $1.9(1.1)$ \\
\hline \multirow[t]{2}{*}{ CYFRA21-1 $(\mu \mathrm{g} / \mathrm{l})$} & Mean (SD) & $14.2(26.0)$ & $16.0(27.4)$ & $1.7(0.8)$ & $2.3(2.3)$ & $1.8(1.1)$ & $1.8(0.9)$ \\
\hline & Median (IQR) & $4.2(9.9)^{\mathrm{a}}$ & $5.5(14.7)^{\mathrm{b}, \mathrm{c}}$ & $1.6(0.9)$ & $1.7(1.4)$ & $1.1(1,1)$ & $1.5(1.3)$ \\
\hline \multirow[t]{2}{*}{ CA 19-9 (KU/1) } & Mean (SD) & $2,286(14,497)$ & $2,622(15,511)$ & $15.3(17.4)$ & $19.9(25.8)$ & $14.7(20.0)$ & $12.4(2.8)$ \\
\hline & Median (IQR) & $33.5(160)^{\mathrm{a}}$ & $53(214)^{\mathrm{b}, \mathrm{c}}$ & $7.0(21.0)$ & $11.0(16.0)$ & $10.0(10.0)$ & $8.0(12.0)$ \\
\hline \multirow[t]{2}{*}{ CA 15-3 (KU/1) } & Mean (SD) & $50.4(106)$ & $53.9(113.1)$ & $26.9(15.4)$ & $21.0(11.1)$ & $19.2(9.4)$ & $18.6(7.6)$ \\
\hline & Median (IQR) & $24.0(21.5)^{\mathrm{a}}$ & $25.4(20.9)^{\mathrm{b}}$ & $20.5(48.9)$ & $19.1(12.1)$ & $17.2(11.7)$ & $18.7(9.9)$ \\
\hline \multirow[t]{2}{*}{ CA $125(\mathrm{KU} / \mathrm{l})$} & Mean (SD) & $155(649.1)$ & $172(695)$ & $41.0(50.3)$ & $24.0(33.9)$ & $16.4(14.8)$ & $12.8(7.7)$ \\
\hline & Median (IQR) & $24.5(48.1)^{\mathrm{a}}$ & $25.5(57.1)^{\mathrm{b}}$ & $20.2(40.9)^{\mathrm{e}}$ & $14.8(17.9)^{\mathrm{d}, \mathrm{f}}$ & $12.2(9.5)$ & $9.7(8.9)$ \\
\hline \multirow[t]{2}{*}{$\operatorname{AFP}(\mu \mathrm{g} / \mathrm{l})$} & Mean (SD) & $921(7310)$ & $1,062(7847)$ & $2.2(2.0)$ & $3.1(9,3)$ & $2,6(2.0)$ & $2.3(1.3)$ \\
\hline & Median (IQR) & $2.0(2.0)$ & $2.1(2.1)$ & $1.7(1.0)$ & $2.1(1.9)$ & $2.1(1.7)$ & $2.1(2.2)$ \\
\hline \multirow[t]{2}{*}{ NSE $(\mu \mathrm{g} / \mathrm{l})$} & Mean (SD) & $32.0(76.1)$ & $34.6(83.6)$ & $19.9(17.6)$ & $11.1(3.8)$ & $11.0(4.3)$ & $10.3(1.6)$ \\
\hline & Median (IQR) & $12.8(15.3)^{\mathrm{a}}$ & $12.8(16.1)^{\mathrm{b}}$ & $13,5(14.3)^{\mathrm{e}}$ & $10.5(3.5)$ & $10.1(4.2)$ & $10.4(2.5)$ \\
\hline \multirow[t]{2}{*}{ PSA $(\mu \mathrm{g} / 1)$} & Mean (SD) & $29.7(170.5)$ & $33.1(180)$ & $1.2(0.9)$ & $2.6(3.4)$ & $2.2(3.0)$ & $1.8(1.6)$ \\
\hline & Median (IQR) & $1.4(2.3)$ & $1.4(2.6)$ & $1.5(1.7)$ & $1.3(2.7)$ & $1.2(1.9)$ & $1.4(1.9)$ \\
\hline
\end{tabular}

aSignificant difference $(p<0.02)$ between neoplasia and benign disease; ${ }^{b}$ Significant difference $(p<0.05)$ between epithelial neoplasia and benign disease; 'Significant difference $(p<0.001)$ between neoplasia epithelial and no epithelial; dSignificant difference $(p<0.05)$ between organic no neoplastic and psychiatric; eSignificant difference $(p<0.03)$ between neoplasia non-epithelial and benign disease; f Significant difference $(p<0.021)$ between organic no neoplastic and unknown origin.

Table III. Diagnostic accuracy of tumour markers based on the cut-off of upper reference interval.

\begin{tabular}{|c|c|c|c|c|c|c|c|}
\hline Tumour markers & $\begin{array}{l}\text { Sensitivity } \\
(95 \% \mathrm{CI})\end{array}$ & $\begin{array}{l}\text { Specificity } \\
(95 \% \mathrm{CI})\end{array}$ & $\begin{array}{c}\mathrm{NPV} \\
(95 \% \mathrm{CI})\end{array}$ & $\begin{array}{c}\text { PPV } \\
(95 \% \mathrm{CI})\end{array}$ & $\begin{array}{c}\text { NLR } \\
(95 \% \mathrm{CI})\end{array}$ & $\begin{array}{c}\text { PLR } \\
(95 \% \mathrm{CI})\end{array}$ & $\begin{array}{l}\text { Accuracy } \\
(95 \% \mathrm{CI})\end{array}$ \\
\hline CEA $(5 \mu \mathrm{g} / \mathrm{l})(\mathrm{n}=600)$ & $\begin{array}{c}50.7 \% \\
(42.3-59.1)\end{array}$ & $\begin{array}{c}86.5 \% \\
(82.9-89.4)\end{array}$ & $\begin{array}{c}85.0 \% \\
(81.4-88.1)\end{array}$ & $\begin{array}{c}53.7 \% \\
(44.9-62.3)\end{array}$ & $\begin{array}{c}0.6 \\
(0.5-0.7)\end{array}$ & $\begin{array}{c}3.7 \\
(2.8-5.0)\end{array}$ & $\begin{array}{c}78.0 \% \\
(74.4-81.2)\end{array}$ \\
\hline $\begin{array}{l}\text { CYFRA 21-1 }(3.3 \mu \mathrm{g} / \mathrm{l}) \\
(\mathrm{n}=567)\end{array}$ & $\begin{array}{c}55.0 \% \\
(46.0-63.6)\end{array}$ & $\begin{array}{c}87.4 \% \\
(83.8-90.2)\end{array}$ & $\begin{array}{c}86.6 \% \\
(83.0-89.5)\end{array}$ & $\begin{array}{c}56.7 \% \\
(46.0-63.5)\end{array}$ & $\begin{array}{c}0.5 \\
(0.4-0.6)\end{array}$ & $\begin{array}{c}4.4 \\
(3.3-5.8)\end{array}$ & $\begin{array}{c}79.9 \% \\
(76.3-83.1)\end{array}$ \\
\hline CA19-9 $(37 \mathrm{U} / \mathrm{ml})(\mathrm{n}=572)$ & $\begin{array}{c}46.9 \% \\
(38.3-55.8)\end{array}$ & $\begin{array}{c}90.2 \% \\
(87.0-92.8)\end{array}$ & $\begin{array}{c}85.0 \% \\
(81.4-88.1)\end{array}$ & $\begin{array}{c}59.0 \% \\
(49.0-68.4)\end{array}$ & $\begin{array}{c}0.59 \\
(0.5-0.7)\end{array}$ & $\begin{array}{c}4.81 \\
(3.4-6.7)\end{array}$ & $\begin{array}{c}80.2 \% \\
(76.6-83.5)\end{array}$ \\
\hline $\operatorname{NSE}(17 \mu \mathrm{g} / \mathrm{l})(\mathrm{n}=404)$ & $\begin{array}{c}29.2 \% \\
(20.6-39.5)\end{array}$ & $\begin{array}{c}96.7 \% \\
(93.8-98.3)\end{array}$ & $\begin{array}{c}73.7 \% \\
(56.6-86.0)\end{array}$ & $\begin{array}{c}81.1 \% \\
(76.6-84.9)\end{array}$ & $\begin{array}{c}0.73 \\
(0.6-0.8)\end{array}$ & $\begin{array}{c}8.78 \\
(4.4-17.4)\end{array}$ & $\begin{array}{c}80.3 \% \\
(76.0-84.1)\end{array}$ \\
\hline CA15-3 $(30 \mathrm{U} / \mathrm{ml})(\mathrm{n}=560)$ & $\begin{array}{c}38.3 \% \\
(30.0-47.3)\end{array}$ & $\begin{array}{c}89.3 \% \\
(85.9-92.0)\end{array}$ & $\begin{array}{c}77.4 \% \\
(73.6-80.8)\end{array}$ & $\begin{array}{c}52.1 \% \\
(41.6-62.4)\end{array}$ & $\begin{array}{c}0.7 \\
(0.6-0.8)\end{array}$ & $\begin{array}{c}3.6 \\
(2.5-5.1)\end{array}$ & $\begin{array}{c}77.4 \% \\
(73.6-80.8)\end{array}$ \\
\hline CA125 (35 U/ml) $(\mathrm{n}=549)$ & $\begin{array}{c}43.2 \% \\
(34.7-52.1)\end{array}$ & $\begin{array}{c}83.0 \% \\
(79.1-86.3)\end{array}$ & $\begin{array}{c}85.7 \% \\
(82.0-88.8)\end{array}$ & $\begin{array}{c}43.2 \% \\
(34.7-52.1)\end{array}$ & $\begin{array}{c}0.62 \\
(0.5-0.7)\end{array}$ & $\begin{array}{c}2.85 \\
(2.2-3.8)\end{array}$ & $\begin{array}{c}75.7 \% \\
(71.8-79.1)\end{array}$ \\
\hline $\operatorname{AFP}(10 \mu \mathrm{g} / 1)(\mathrm{n}=547)$ & $\begin{array}{c}6.7 \% \\
(3.2-13.1)\end{array}$ & $\begin{array}{c}99.1 \% \\
(97.4-99.7)\end{array}$ & $\begin{array}{c}79.1 \% \\
(75.3-82.4)\end{array}$ & $\begin{array}{c}66.7 \% \\
(35.4-82.4)\end{array}$ & $\begin{array}{c}0.94 \\
(0.90-0.99)\end{array}$ & $\begin{array}{c}7.12 \\
(2.2-23.2)\end{array}$ & $\begin{array}{c}78.8 \% \\
(75.1-82.1)\end{array}$ \\
\hline $\operatorname{PSA}(4 \mu \mathrm{g} / \mathrm{l})(\mathrm{n}=254)$ & $\begin{array}{c}14.6 \% \\
(8.1-24.6)\end{array}$ & $\begin{array}{c}82.6 \% \\
(75.9-87.7)\end{array}$ & $\begin{array}{c}67.0 \% \\
(60.1-73.2)\end{array}$ & $\begin{array}{c}28.6 \% \\
(16.2-44.8)\end{array}$ & $\begin{array}{c}1.03 \\
(0.9-1.1)\end{array}$ & $\begin{array}{c}0.84 \\
(0.45-1.55)\end{array}$ & $\begin{array}{c}60.6 \% \\
(54.3-66.6)\end{array}$ \\
\hline $\begin{array}{l}\text { At least one TM }>\text { cut-off } \\
(\mathrm{n}=606)\end{array}$ & $\begin{array}{c}91.6 \% \\
(85.5-95.4)\end{array}$ & $\begin{array}{c}55.9 \% \\
(51.3-60.5)\end{array}$ & $\begin{array}{c}95.7 \% \\
(92.2-97.6)\end{array}$ & $\begin{array}{c}39.1 \% \\
(33.9-44.6)\end{array}$ & $\begin{array}{c}0.15 \\
(0.09-0.26)\end{array}$ & $\begin{array}{c}2.1 \\
(1.9-2.3)\end{array}$ & $\begin{array}{c}64.4 \% \\
(60.4-68.2)\end{array}$ \\
\hline
\end{tabular}

95\% CI: 95\% Confidence interval; NPV: negative predictive value; PPV: positive predictive value; NLR: negative likelihood ratio; PLR: positive likelihood ratio.

cut-off point, a sensitivity of $64.3 \%$, a specificity of $98.3 \%$, a PPV of $92 \%$, and a PNV of $89.9 \%$ were obtained. In order to assess the cancer risk, we used two cut-off points (upper reference limit and the high cut-off point) which allowed us to classify patients into three groups with low, moderate $(\mathrm{OR}=4.3)$ and very high $(\mathrm{OR}=245)$ cancer risk. 
Trape et al: Tumor Markers in Isolated Involuntary Weight Loss

Table IV. Diagnostic accuracy of tumour markers based on the high cut-off proposed by Molina et al. (18).

\begin{tabular}{|c|c|c|c|c|c|c|c|}
\hline Tumour markers & $\begin{array}{l}\text { Sensitivity } \\
(95 \% \mathrm{CI})\end{array}$ & $\begin{array}{l}\text { Specificity } \\
(95 \% \mathrm{CI})\end{array}$ & $\begin{array}{c}\text { NPV } \\
(95 \% \mathrm{CI})\end{array}$ & $\begin{array}{c}\text { PPV } \\
(95 \% \mathrm{CI})\end{array}$ & $\begin{array}{c}\text { NLR } \\
(95 \% \mathrm{CI})\end{array}$ & $\begin{array}{c}\text { PLR } \\
(95 \% \mathrm{CI})\end{array}$ & $\begin{array}{l}\text { Accuracy } \\
(95 \% \mathrm{CI})\end{array}$ \\
\hline CEA $(15 \mu \mathrm{g} / 1)(\mathrm{n}=600)$ & $\begin{array}{c}31.0 \% \\
(23.6-39.4)\end{array}$ & $\begin{array}{c}99.6 \% \\
(98.3-99.9)\end{array}$ & $\begin{array}{c}82.3 \% \\
(78.8-85.4)\end{array}$ & $\begin{array}{c}95.7 \% \\
(83.4-99.2)\end{array}$ & $\begin{array}{c}0.69 \\
(0.62-0.77)\end{array}$ & $\begin{array}{c}71.1 \\
(17.5-289.7)\end{array}$ & $\begin{array}{c}83.3 \% \\
(80.0-86.2)\end{array}$ \\
\hline $\begin{array}{l}\text { CYFRA 21-1 }(7.5 \mu \mathrm{g} / \mathrm{l}) \\
(\mathrm{n}=567)\end{array}$ & $\begin{array}{c}38.9 \% \\
(30.7-47.9)\end{array}$ & $\begin{array}{c}99.1 \% \\
(97.5-99.7)\end{array}$ & $\begin{array}{c}84.4 \% \\
(80.9-87.3)\end{array}$ & $\begin{array}{c}92.7 \% \\
(81.6-97.6)\end{array}$ & $\begin{array}{c}0.62 \\
(0.54-0.71)\end{array}$ & $\begin{array}{c}42.4 \\
(15.6-115.2)\end{array}$ & $\begin{array}{c}85.2 \% \\
(81.9-88.0)\end{array}$ \\
\hline CA19-9 (200 U/ml) (n=572) & $\begin{array}{c}22.7 \% \\
(16.1-31.0)\end{array}$ & $\begin{array}{c}100 \% \\
(98.9-100)\end{array}$ & $\begin{array}{c}81.2 \% \\
(77.6-84.3)\end{array}$ & $\begin{array}{c}100 \% \\
(85.9-100)\end{array}$ & $\begin{array}{c}0.77 \\
0.70-0.85)\end{array}$ & $>9,999$ & $\begin{array}{c}82.1 \% \\
(78.7-85.1)\end{array}$ \\
\hline $\operatorname{NSE}(45 \mu \mathrm{g} / 1)(\mathrm{n}=404)$ & $\begin{array}{c}14.6 \% \\
(8.5-23.6)\end{array}$ & $\begin{array}{c}100 \% \\
98.4-100)\end{array}$ & $\begin{array}{c}78.6 \% \\
(74.1-82.5)\end{array}$ & $\begin{array}{c}100 \\
(98.4-100)\end{array}$ & $\begin{array}{c}0.85 \\
(0.79-0.93)\end{array}$ & $>9,999$ & $\begin{array}{c}79.4 \% \\
(75.0-83.2)\end{array}$ \\
\hline CA125 (350 U/ml) $(\mathrm{n}=549)$ & $\begin{array}{c}7.0 \% \\
(3.5-13.3)\end{array}$ & $\begin{array}{c}100 \% \\
(98.9-100)\end{array}$ & $\begin{array}{c}77.9 \% \\
(74.1-81.3)\end{array}$ & $\begin{array}{c}100 \% \\
(62.3-100)\end{array}$ & $\begin{array}{c}0.93 \\
(0.89-0.98)\end{array}$ & $>9,999$ & $\begin{array}{c}78.6 \% \\
(75.0-81.9)\end{array}$ \\
\hline CA15-3 (100 U/ml) $(\mathrm{n}=560)$ & $\begin{array}{c}6.1 \% \\
(2.85-12.0)\end{array}$ & $\begin{array}{c}99.8 \% \\
(98.5-99.9)\end{array}$ & $\begin{array}{c}77.5 \% \\
(73.7-80.9)\end{array}$ & $\begin{array}{c}88.9 \% \\
(50.7-99.4)\end{array}$ & $\begin{array}{c}0.94 \\
(0.9-0.98)\end{array}$ & $\begin{array}{c}25.9 \\
(3.3-205.5)\end{array}$ & $\begin{array}{c}77.7 \% \\
(74.0-81.0)\end{array}$ \\
\hline AFP $(40 \mu \mathrm{g} / \mathrm{l})(\mathrm{n}=547)$ & $\begin{array}{c}5.0 \% \\
(2.0-11.0)\end{array}$ & $\begin{array}{c}99.7 \% \\
(97.9-99.9)\end{array}$ & $\begin{array}{c}72.5 \% \\
(67.9-76.7)\end{array}$ & $\begin{array}{c}85.7 \% \\
(42.0-99.2)\end{array}$ & $\begin{array}{c}0.95 \\
(0.91-0.99)\end{array}$ & $\begin{array}{c}15.1 \\
(1.8-124.1)\end{array}$ & $\begin{array}{c}79.0 \% \\
(75.3-82.3)\end{array}$ \\
\hline PSA $(30 \mu \mathrm{g} / \mathrm{l})(\mathrm{n}=254)$ & $\begin{array}{c}3.7 \% \\
(1.0-11.1)\end{array}$ & $\begin{array}{c}100 \% \\
(97.3-100)\end{array}$ & $\begin{array}{c}68.5 \% \\
(62.5-74.3)\end{array}$ & $\begin{array}{c}100 \% \\
(31.0-100)\end{array}$ & $\begin{array}{c}0.96 \\
(0.92-1.0)\end{array}$ & $>9,999$ & $\begin{array}{c}69.0 \% \\
(62.9-74.6)\end{array}$ \\
\hline $\begin{array}{l}\text { At least one TM>cut-off } \\
(\mathrm{n}=606)\end{array}$ & $\begin{array}{c}64.3 \% \\
(55.8-72.0)\end{array}$ & $\begin{array}{c}98.3 \% \\
(96.5-99.2)\end{array}$ & $\begin{array}{c}89.9 \% \\
(86.9-92.4)\end{array}$ & $\begin{array}{c}92.0 \% \\
(84.4-96.3)\end{array}$ & $\begin{array}{c}0.36 \\
(0.29-0.45)\end{array}$ & $\begin{array}{c}37.3 \\
(18.6-75.0)\end{array}$ & $\begin{array}{c}90.3 \% \\
(87.6-92.4)\end{array}$ \\
\hline
\end{tabular}

95\%CI: 95\% Confidence interval; NPV: negative predictive value; PPV: positive predictive value; NLR: negative likelihood ratio; PLR: positive likelihood ratio.

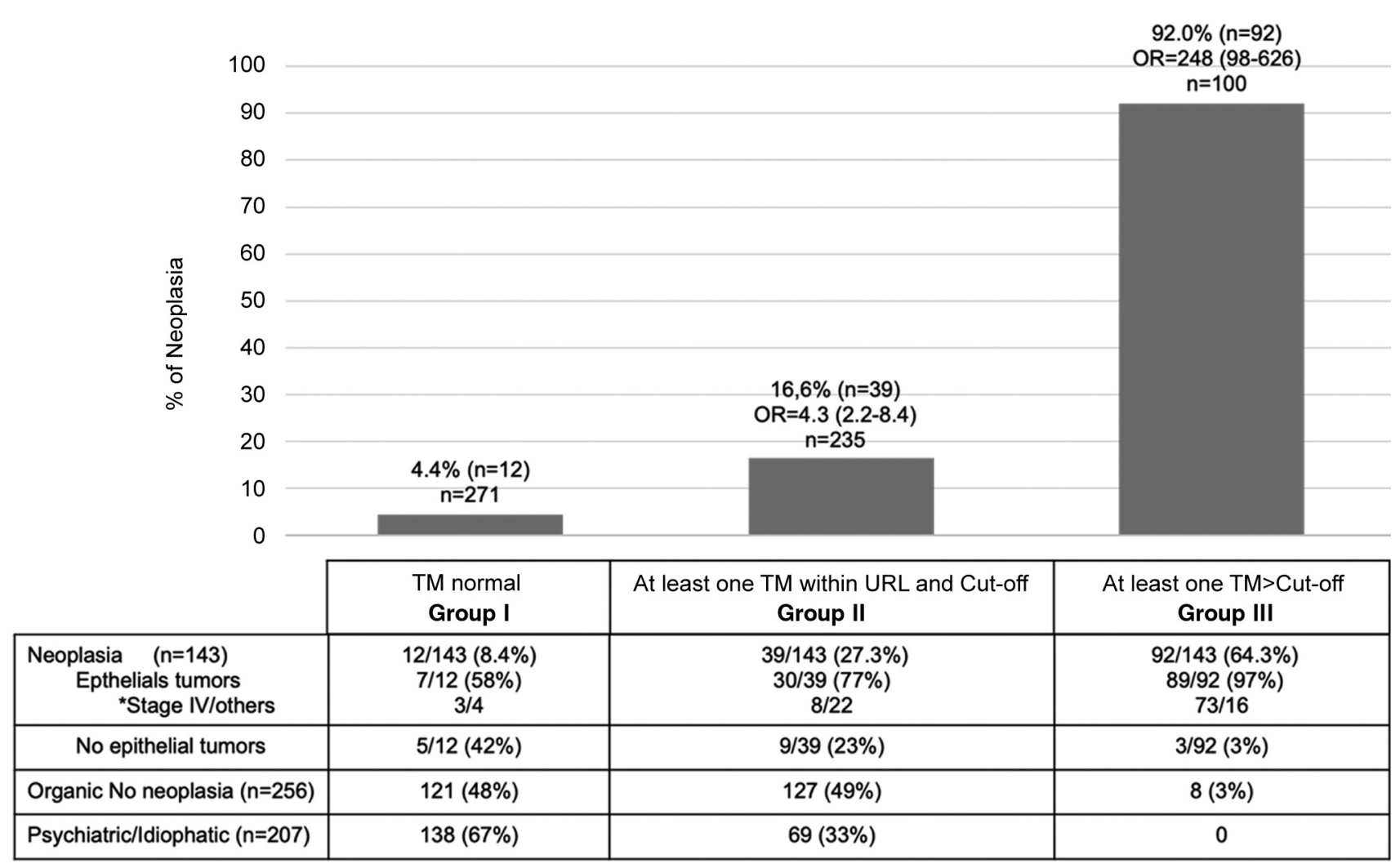

*Only Epithelial tumors

Figure 2. Distribution of type of disease according to the group of tumour markers. 
Although some investigators advocate the measurement of TMs for cancer diagnosis, it is not currently included in the guidelines produced by scientific societies. Kilpatrick (19) reported falsely elevated results of certain markers in patients with cirrhosis and ascites, a situation that required the performance of other tests to rule out the suspicion of cancer. These authors also warned that results within the reference limit give a false sense of security, dispelling the suspicion of cancer and delaying the diagnosis. Sturgeon (20) also argued against the use of TM panels because of the limited benefit they provide in individual patients. In recent years, however, some studies have shown that in some types of cancer, a test for a combination of TMs can improve individual assessment. In 2016, Molina (21) created a panel of six TMs in patients with suspected lung cancer, which had a higher diagnostic accuracy than the same TMs considered individually. This panel was able to distinguish small cell lung cancer from non-small cell lung cancer based on the NSE and ProGRP markers. Blanco-Prieto (22) using two conventional TMs and other biological markers (CEA, CYFRA 21-1, sCD26, calprotectin, MMP1, MMP7 and MMP9 EGF) improved the clinical model, obtaining a sensitivity of $95 \%$ and a specificity of $43.6 \%$.

In 2015, using three TMs (CEA, CYFRA 21-1, and CA199), our group (23) studied 234 patients with symptoms and signs of cancer using two cut-off points, one for patients without liver and kidney involvement and a higher one for those who had either renal involvement (creatinine $>1.5$ $\mathrm{mg} / \mathrm{dl}$ ) and/or liver involvement (bilirubin $>1.5 \mathrm{mg} / \mathrm{dl}$ ), obtaining a sensitivity of $54 \%$ and a specificity of $100 \%$.

Molina et al. 2012 (18) evaluated the diagnostic capacity of 10 TMs (CEA, CYFRA 21-1, CA15-3 CA19-9, CA72-4, CA125, NSE, SCC, AFP and PSA) in the differential diagnosis between benign and malignant disease, in 2,711 patients with signs or symptoms of cancer. These authors applied two cutoff points: one above the upper reference limit (the one used in the present study) to increase the specificity, and another for patients with clinicopathological alterations that raised TM concentration well above the upper reference limit, such as renal failure, jaundice, effusions and dermatological diseases, and obtained a sensitivity of $67 \%$ and a specificity of $98 \%$. In 2021, the same group (24) added ProGRP to the panel and with a higher case volume (4,776 patients) obtained a sensitivity of $72.2 \%$ and a specificity of $98 \%$.

The present study focused on outpatients with IIWL as the only sign of cancer. The same cut-off point as in studies of Molina (2012) and Bosch (2021) (24) was used because none of the patients had serous effusions, dermatological disease, kidney failure or jaundice. The sensitivity was similar to that obtained in previous studies; the higher the number of TMs, the greater the sensitivity, with a specificity around $98 \%$.

The presence of IIWL may raise the suspicion of cancer, but it is by no means definitive. Some authors have searched for predictive models of cancer risk on the basis of laboratory tests. When only biochemical and hematologic parameters are used, the capacity for predicting cancer is moderate. Baicus et al. (25) found ORs for age $>60$ years, albumin $>3.5 \mathrm{~g} / \mathrm{dl}$ and alkaline phosphatase $<104 \mathrm{U} / 1$ of 5.1 (1.88-15.02), 2.44 (1.145.23), and 2.67 (1.23-5.8) respectively, Casarrubias-Ramírez et al. (26) found ORs of 3.9 (1.7-8.9) for albumin $>3.5 \mathrm{~g} / \mathrm{dl}$, and of 4.7 (1.3-17.5) for alkaline phosphatase $>300 \mathrm{U} / 1$. The use of TMs has obtained better classifications: Torné-Cachot (27) found TMs to be the only factors that maintained their power for predicting cancer in a multivariate analysis, with ORs of $2.38(95 \% \mathrm{CI}=1.17-4.8)$ for a one TM above cut-of and of $6.51(95 \% \mathrm{CI}=2.62-16.13)$ for two TMs above cut-off. In our study, we found an OR of $4.3(95 \% \mathrm{CI}=2.2-8.4)$ for at least one marker between the upper reference limit and the high cut-off, and one of 245 (95\% CI=98-626) for patients with at least one marker above the high cut-off. Considering our classification into three groups, the rates of tumors were $4.4 \%(n=12)$ in the low-risk group, $16.6 \%(n=39)$ in the moderate-risk group and $92 \%(n=92)$ in the high-risk group. In group I the NPV was higher than $95 \%$, whereas in group III the PPV was 92\%, which allows an accurate evaluation of the cancer risk and the selection of the most appropriate additional tests in order to obtain a prompt diagnosis.

Although the markers were determined before obtaining the final diagnosis, the main limitation of this study was that it was performed at a single centre. The results should now be validated in multicentre studies involving laboratory medicine specialists and clinicians and using feasible end points, such as less invasive and less expensive diagnostic procedures.

\section{Conclusion}

The determination of TMs for the classification of patients with IIWL according to cancer risk allows for a better selection of complementary tests to obtain the final diagnosis.

\section{Conflicts of Interest}

The Authors declare no conflicts of interest in relation to this study.

\section{Authors' Contributions}

Designed research/study: JT, JA, JO, AS-J, DR. Performed research: JT, JA, JO, MV. Collected data: MB, AAb, MS, CF, CG-F, MV, LM, OE-B, RO, EG-G. Analyzed data: Aar. Wrote the paper: JT, JA.

\section{References}

1 Bosch X, Monclús E, Escoda O, Guerra-García M, Moreno P, Guasch N and López-Soto A: Unintentional weight loss: Clinical characteristics and outcomes in a prospective cohort of 2677 patients. PLoS One 12(4): e0175125, 2017. PMID: 28388637. DOI: $10.1371 /$ journal.pone.0175125 
2 Schmidt-Hansen M, Berendse S and Hamilton W: Symptoms of pancreatic cancer in primary care: a systematic review. Pancreas 45(6): 814-818, 2016. PMID: 26495795. DOI: 10.1097/MPA. 0000000000000527

3 Mondello $\mathrm{P}$, Mian M, Aloisi C, Famà F, Mondello S and Pitini V: Cancer cachexia syndrome: pathogenesis, diagnosis, and new therapeutic options. Nutr Cancer 67(1): 12-26, 2015. PMID: 25513730. DOI: $10.1080 / 01635581.2015 .976318$

4 Schmidt SF, Rohm M, Herzig S and Berriel Diaz M: Cancer cachexia: More than skeletal muscle wasting. Trends Cancer 4(12): 849-860, 2018. PMID: 30470306. DOI: 10.1016/j.trecan. 2018.10.001

5 Baizabal-Carvallo JF and Jankovic J: Autoimmune and paraneoplastic movement disorders: An update. J Neurol Sci 385: 175-184, 2018. PMID: 29406902. DOI: 10.1016/j.jns.2017.12.035

6 Pelosof LC and Gerber DE: Paraneoplastic syndromes: an approach to diagnosis and treatment. Mayo Clin Proc 85(9): 838-854, 2010. PMID: 20810794. DOI: $10.4065 / \mathrm{mcp} .2010 .0099$

7 Bosch X, Foix A, Jordan A, Coca A and López-Soto A: Outpatient Quick Diagnosis Units for the evaluation of suspected severe diseases: an observational, descriptive study. Clinics (Sao Paulo) 66(5): 737-741, 2011. PMID: 21789373. DOI: 10.1590/ s1807-59322011000500005

8 Sanclemente-Ansó C, Bosch X, Salazar A, Moreno R, Capdevila C, Rosón B and Corbella X: Cost-minimization analysis favors outpatient quick diagnosis unit over hospitalization for the diagnosis of potentially serious diseases. Eur J Intern Med 30: 11-17, 2016. PMID: 26944565. DOI: 10.1016/j.ejim.2015.12.015

9 Montori-Palacín E, Prieto-González S, Carrasco-Miserachs I, AltesCapella J, Compta Y, López-Soto A and Bosch X: Quick outpatient diagnosis in small district or general tertiary hospitals: A comparative observational study. Medicine (Baltimore) 96(22): e6886, 2017. PMID: 28562538. DOI: 10.1097/MD.0000000000006886

10 Wang X, Zhang Y, Sun L, Wang S, Nie J, Zhao W and Zheng G: Evaluation of the clinical application of multiple tumor marker protein chip in the diagnostic of lung cancer. J Clin Lab Anal 32(8): e22565, 2018. PMID: 29736949. DOI: 10.1002/jcla.22565

11 Duffy MJ and O'Byrne K: Tissue and blood biomarkers in lung cancer: a review. Adv Clin Chem 86: 1-21, 2018. PMID: 30144837. DOI: $10.1016 /$ bs.acc. 2018.05 .001

12 Lee JH: The feasibility of serum multiple tumor markers test between patients with primary pancreatic cancer and those with benign pancreatic cystic disease. Clin Lab 65(10), 2019. PMID: 31625360. DOI: 10.7754/Clin.Lab.2019.190222

13 Trapé J, Botargues JM, Porta F, Ricós C, Badal JM, Salinas R, Sala $M$ and Roca A: Reference change value for alpha-fetoprotein and its application in early detection of hepatocellular carcinoma in patients with hepatic disease. Clin Chem 49(7): 1209-1211, 2003. PMID: 12816928 . DOI: $10.1373 / 49.7 .1209$

14 Chen C, Chen Q, Zhao Q, Liu M and Guo J: Value of combined detection of serum CEA, CA72-4, CA19-9, CA15-3 and CA125 in the diagnosis of gastric cancer. Ann Clin Lab Sci 47(3): 260-263, 2017. PMID: 28667025.

15 Wang W, Xu X, Tian B, Wang Y, Du L, Sun T, Shi Y, Zhao X and Jing J: The diagnostic value of serum tumor markers CEA, CA19-9, CA125, CA15-3, and TPS in metastatic breast cancer. Clin Chim Acta 470: 51-55, 2017. PMID: 28457854. DOI: 10.1016/j.cca.2017.04.023

16 Trapé J, Filella X, Alsina-Donadeu M, Juan-Pereira L, BoschFerrer Á, Rigo-Bonnin R and Oncology Section of the Catalan
Association of Clinical Laboratory Science: Increased plasma concentrations of tumour markers in the absence of neoplasia. Clin Chem Lab Med 49(10): 1605-1620, 2011. PMID: 21892908. DOI: 10.1515/CCLM.2011.694

17 Marton KI, Sox HC Jr and Krupp JR: Involuntary weight loss: diagnostic and prognostic significance. Ann Intern Med 95(5): 568-574, 1981. PMID: 7294545. DOI: 10.7326/0003-4819-95$5-568$

18 Molina R, Bosch X, Auge JM, Filella X, Escudero JM, Molina V, Solé M and López-Soto A: Utility of serum tumor markers as an aid in the differential diagnosis of patients with clinical suspicion of cancer and in patients with cancer of unknown primary site. Tumour Biol 33(2): 463-474, 2012. PMID: 22161237. DOI: $10.1007 / \mathrm{s} 13277-011-0275-1$

19 Kilpatrick ES and Lind MJ: Appropriate requesting of serum tumour markers. BMJ 339: b3111, 2009. PMID: 19773324. DOI: 10.1136/bmj.b3111

20 Sturgeon CM, Lai LC and Duffy MJ: Serum tumour markers: how to order and interpret them. BMJ 339: b3527, 2009. PMID: 19773328. DOI: 10.1136/bmj.b3527

21 Molina R, Marrades RM, Augé JM, Escudero JM, Viñolas N, Reguart N, Ramirez J, Filella X, Molins L and Agustí A: Assessment of a combined panel of six serum tumor markers for lung cancer. Am J Respir Crit Care Med 193(4): 427-437, 2016. PMID: 26465739. DOI: 10.1164/rccm.201404-0603OC

22 Blanco-Prieto S, De Chiara L, Rodríguez-Girondo M, VázquezIglesias L, Rodríguez-Berrocal FJ, Fernández-Villar A, BotanaRial MI and de la Cadena MP: Highly sensitive marker panel for guidance in lung cancer rapid diagnostic units. Sci Rep 7: 41151, 2017. PMID: 28117344. DOI: 10.1038/srep41151

23 Trapé J, Sala M, Franquesa F, Ordeig JM, Soler-Bel JM, Bustamante E, Pérez R, Aligué J, Montesinos J, Arnau A and Ordeig-Villanueva R: Clinical utility of determining tumor markers in patients with signs and symptoms of cancer. Clin Chem Lab Med 53(3): 485-491, 2015. PMID: 25274947. DOI: 10.1515/cclm-2014-0410

24 Bosch X, Molina R, Marrades R, Augé JM, Pellicé M and López-Soto A: Tumour markers with clinically controlled cutoffs for suspected cancer. Eur J Clin Invest 51(7): e13523, 2021. PMID: 33594702. DOI: 10.1111/eci.13523

25 Baicus C, Rimbas M, Baicus A, Caraiola S and Grupul de Studiu al Scaderii Ponderale Involuntare: Cancer and involuntary weight loss: failure to validate a prediction score. PLoS One 9(4): e95286, 2014. PMID: 24762986. DOI: 10.1371/journal. pone.0095286

26 Casarrubias-Ramírez M, López-Martínez JE, Pineda-Galindo LF, Carrillo-González AL, Zavaleta-Ramírez E and Iniestra-Flores F: Validation of a predictive index of neoplasia in unexplained weight loss. Rev Med Inst Mex Seguro Soc 57(3): 133-139, 2019. PMID: 31995335.

27 Torné Cachot J, Baucells Azcona JM, Blanch Falp J, García Pont $\mathrm{J}$ and Camell Ilari $\mathrm{H}$ : Isolated involuntary weight loss: Epidemiology and predictive factors of malignancy. Med Clin (Barc) 152(10): 384-390, 2019. PMID: 30297253. DOI: 10.1016/j.medcli.2018.07.010
Received July 15, 2021

Revised August 7, 2021

Accepted August 11, 2021 